\title{
YAP as a key regulator of adipo-osteogenic differentiation in human MSCs
}

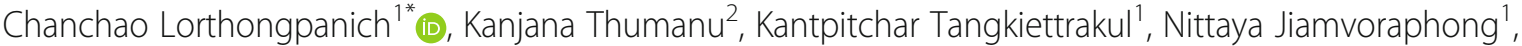 \\ Chuti Laowtammathron', Nattaya Damkham,3, Yaowalak U-pratya ${ }^{1,3}$ and Surapol Issaragrisil ${ }^{1,4,5}$
}

\begin{abstract}
Background: Mesenchymal stem cells (MSCs) are multipotent stem cells that are able to differentiate into several cell types, including cartilage, fat, and bone. As a common progenitor, MSC differentiation has to be tightly regulated to maintain the balance of their differentiation commitment. It has been reported that the decision process of MSCs into fat and bone cells is competing and reciprocal. Several factors have been suggested as critical factors that affect adipo-osteogenic decision, including melatonin and smad4. Yes-associated protein (YAP) is an important effector protein in the Hippo signaling pathway that acts as a transcriptional regulator by activating the transcription of the genes involved in cell proliferation and anti-apoptosis. The non-canonical role of YAP in regulating bone homeostasis by promoting osteogenesis and suppressing adipogenesis was recently demonstrated in a mouse model. However, it is unclear whether YAP is also crucial for modulating human MSC differentiation to fat and bone.
\end{abstract}

Methods: The expression level of YAP during MSC differentiation was modulated using pharmaceutical molecule and genetic experiments through gain- and loss-of-function approaches.

Results: We demonstrated for the first time that YAP has a non-canonical role in regulating the balance of adipoosteogenic differentiation of human MSCs. The result from synchrotron radiation-based Fourier transform infrared (FTIR) microspectroscopy showed unique metabolic fingerprints generated from YAP-targeted differentiated cells that were clearly distinguished from non-manipulated control.

Conclusions: These results, thus, identify YAP as an important effector protein that regulates human MSC differentiation to fat and bone and suggests the use of FTIR microspectroscopy as a promising technique in stem cell research.

Keywords: FTIR microspectroscopy, Dobutamine, LPA, MSCs, Adipo-osteogenic balance, YAP, Hippo pathway

\section{Background}

Mesenchymal stem cells/stromal cells (MSCs) are a specialized population of progenitor cells that can be isolated from various adult or fetal tissues and membranes [1]. Due to their hypoimmunogenic or immune privilege, MSCs have been used in regenerative therapy, especially for transplantation across major histocompatibility barriers $[2,3]$. MSCs are multipotent stem cells that are able to differentiate into several cell types, including osteoblasts, chondrocytes, adipocytes, and hematopoietic stem

\footnotetext{
* Correspondence: chanchao.lor@mahidol.ac.th

${ }^{1}$ Siriraj Center of Excellence for Stem Cell Research, Department of Medicine, Faculty of Medicine Siriraj Hospital, Mahidol University, 2 Wanglang Road, Bangkoknoi, Bangkok 10700, Thailand

Full list of author information is available at the end of the article
}

cell-supportive stroma. As a common progenitor, MSCs have to maintain a delicate balance for their differentiation commitment, especially for differentiation to fat and bone [4]. It has been demonstrated that bone induction factors, such as RUNX family transcription factor 2 (Runx2), inhibit adipogenesis, whereas peroxisome proliferatoractivated receptor $\gamma$ (PPAR $\gamma$ ) stimulated adipogenesis and inhibited osteogenesis [5]. Bone loss has also been observed in obese mice, rats, and humans [6-8]. Several external cues contribute to the bias of adipo-osteogenic differentiation of MSCs, including chemical [9, 10], physical [11, 12], and biological factors, such as aging/metabolism [13]. These factors trigger different signaling pathways and activate various transcription factors that 
guide MSCs to commit to their differentiation fate [14]. The factors affecting the adipo-osteogenic decision of MSCs are reviewed in detail elsewhere [4].

It was recently observed that activation of the beta- 1 adrenergic signaling pathway by dobutamine hydrochloride (DH), a beta-1 adrenergic agonist, contributes to postmenopausal and age-related bone loss, while blocking of beta-1 adrenergic signaling showed favorable effects on bone turnover [15]. However, the insight molecular mechanism for these phenomena has not been described. Bao and colleagues showed that $\mathrm{DH}$ is able to attenuate yes-associated protein (YAP), which is a transcriptional coactivator that is negatively regulated by the Hippo signaling pathway, by inhibiting its nuclear translocation [16]. YAP and transcriptional co-activator with PDZ-binding motif (TAZ), also known as WW domain-containing transcriptional regulator 1 (WWTR1), were recently suggested as key regulators of bone homeostasis in mice by promoting osteogenesis and suppressing adipogenesis via the Smad4 or beta-catenin signaling pathway [17-19]. However, whether or not YAP plays a role in controlling the adipo-osteogenic balance of human MSCs has never been reported.

Lysophosphatidic acid (LPA) is a phospholipid derivative that can act as a signaling molecule to activate $G$ protein-coupled receptors that are known to regulate cell proliferation and migration. Recent experiments suggested that the Hippo-YAP/TAZ signaling pathway is a downstream target of LPA for regulating cell proliferation and migration. It has been shown that LPA inhibits large tumor suppressor (LATS) kinase, a major core kinase of the Hippo-YAP/TAZ pathway, resulting in activation of YAP transcription coactivator and the expression of its downstream target genes. Though studies in monocytes and CV-1 cells (kidney fibroblast cell line derived from an African green monkey) suggested that LPA could bind to and activate PPARY (which is a transcription factor also known to play a crucial role in adipogenesis), experiments in mouse preadipose cell line (3T3F442A) showed negative effect of LPA on adipogenic differentiation, as demonstrated by a reduction in the PPAR $\gamma$-sensitive genes phosphoenolpyruvate carboxykinase (PEPCK) and adipocyte lipid-binding protein (ALBP) [20]. However, the role of LPA in human MSC differentiation to adipocytes has never been demonstrated.

Synchrotron-based Fourier-transform infrared spectroscopy (FTIR) microspectroscopy is a valuable tool for characterizing and describing the biochemical changes in cells and tissues. The positions of the peaks observed in the FTIR spectra provide significant information relative to the biochemical content of the macromolecules in the cells. Therefore, this information can be used as a fingerprint of the structure and functionality of specimens. The content of these molecules can also be linked to the stage of differentiation and the physiological state of cells [21-25]. FTIR microspectroscopy is a proven label-free method for studying a small sample size with high spatial resolution [26]. With the brightness of SR light, FTIR microspectroscopy can be used to study single cells with a good signal-to-noise ratio [27, 28].

In the present work, we studied the role of YAP in regulating the adipo-osteogenic balance in human MSCs. The expression level of YAP during MSC differentiation was manipulated using the pharmaceutical molecule (DH and LPA to inhibit and activate YAP, respectively) and a genetic approach (Crispr/Cas9 targeting YAP and YAPs5a to inhibit and overexpress YAP, respectively). In addition to other standard procedures that are used to confirm the presence of osteoblasts and adipocytes, FTIR microspectroscopy was used to track the structural changes of nucleic acids, proteins, and lipids in differentiated cells after YAP had been manipulated. The results showed that YAP plays an important role in controlling adipo-osteogenesis in human MSCs. It is clear that YAP is required for MSCs to achieve osteogenic fate, whereas the absence of YAP promotes adipogenic differentiation. This result also strongly suggests the use of FTIR is an effective method for studying cell characteristics of human osteogenesis and adipogenesis.

\section{Materials and methods}

\section{Isolation and culture of hUC-MSCs}

Three umbilical cords (UC) were obtained, cut into small pieces, and incubated with $0.25 \%(\mathrm{w} / \mathrm{v})$ trypsinEDTA (GIBCO ${ }^{\mathrm{m}}$; Invitrogen Corporation, Carlsbad, CA, USA) for $30 \mathrm{~min}$ at $37^{\circ} \mathrm{C}$. Cell suspensions were collected and washed with phosphate-buffered saline (PBS) before being resuspended with culture medium, which consisted of Dulbecco's modified Eagle's medium (DMEM)-low glucose $\left(\mathrm{Gibco}^{\circ}\right)$ supplemented with $10 \%$ fetal bovine serum (FBS; Merck Millipore, Burlington, MA, USA), and plated in culture vessels (Corning, Corning, NY, USA). Cultures were maintained at $37^{\circ} \mathrm{C}$ in a humidified atmosphere containing $5 \% \mathrm{CO}_{2}$. The culture medium was replaced every other day. Adherence cells were treated with $0.05 \%$ Trypsin-EDTA $\left(\mathrm{Gibco}^{\circ}\right)$ and split to a seeding ratio of 5000 cells $/ \mathrm{cm}^{2}$ for expansion.

\section{Immunophenotypical characterization}

Primary culture from UC-MSCs $\left(4 \times 10^{5}\right.$ cells $)$ were resuspended in $50 \mu \mathrm{l}$ of PBS and incubated with $10 \mu \mathrm{l}$ of peridinin-chlorophyll proteins (PerCP), fluorescein isothiocyanate (FITC) or phycoerythrin (PE)-conjugated antibodies against CD45 (BioLegend, San Diego, CA, USA), CD34 (BioLegend), CD73 (BioLegend), CD90 (BioLegend), or CD105 (BioLegend) for $30 \mathrm{~min}$ at $4{ }^{\circ} \mathrm{C}$ in the dark. After washing with PBS, the cells were fixed with $1 \%$ paraformaldehyde (PFA). At least 10,000 labeled cells were acquired and analyzed using flow cytometry 
(FACSCanto $^{\mathrm{TM}}$ or FACSCalibur ${ }^{\mathrm{TM}}$ analyzer; BD Biosciences, San Jose, CA, USA).

\section{Preparation of small molecules}

Lysophosphatidic acid (LPA) and dobutamine hydrochloride (DH) were purchased from Sigma-Aldrich (St. Louis, MO, USA) and prepared as a $20 \mathrm{mmol} / \mathrm{l}$ stock solution in the diluent suggested by the manufacturer's protocol. The final concentration of $10 \mu \mathrm{M}$ of LPA and $20 \mu \mathrm{M}$ of $\mathrm{DH}$ was used as a supplement into osteogenic and adipogenic differentiation media.

\section{Generation of YAP-targeted cells}

Crispr/Cas9 plasmid construct targeting YAP was purchased from GenScript Corporation (Piscataway, NJ, USA). For the preparation of lentivirus, the plasmid was transfected into $4 \times 10^{6} \mathrm{HEK} 293 \mathrm{~T}$ cells in a $100-\mathrm{mm}$ cell culture dish using Lipofectamine 3000 (Life Technologies, Carlsbad, CA, USA). After $24 \mathrm{~h}$, the medium was changed to fresh HEK293T cell medium consisting of DMEM $\left(\mathrm{Gibco}^{\circ}\right)+10 \%$ FBS. Culture media were collected at day 2 after transfection, passed through a 0.45 $\mu \mathrm{M}$ filter (Jet Biofil, Guangzhou, China), and concentrated by transferring the virus-containing supernatant through Amicon Ultra-15 Centrifugal Filter Units (Merck Millipore), followed by centrifugation at $4000 \mathrm{~g}$ for $30 \mathrm{~min}$ at $4{ }^{\circ} \mathrm{C}$. The concentrated virus was collected and added to $5 \times 10^{4} \mathrm{MSCs}$ in the presence of $5 \mu \mathrm{g} / \mathrm{ml}$ polybrene (Sigma-Aldrich). The medium was changed the next day to completed media. The transfected cells were treated with $2 \mu \mathrm{g}$ puromycin for 2 days to eliminate the non-transfected cells before being subjected to osteogenic and adipogenic differentiation.

\section{Generation of YAP-overexpressing cells}

MSCs were transfected with plasmids to promote the overexpression of YAP using 4D nucleofector (Lonza, Basel, Switzerland). At $24 \mathrm{~h}$ after transfection, puromycin $(2 \mu \mathrm{g})$ was added into the culture media for 2 days before the cells were subjected to osteogenic and adipogenic differentiation. Overexpression was confirmed by quantitative real-time polymerase chain reaction (RT-PCR).

\section{Quantitative PCR and data analysis}

Isolated total RNA was reverse-transcribed using a High-Capacity cDNA Reverse Transcription Kit (Applied Biosystems, Foster City, CA, USA). Quantitative RTPCR (qRT-PCR) was performed using Real-Time PCR Master Mix (Applied Biosystems) and the Universal Probe Library (UPL; Roche Life Science, Penzberg, Germany) in a final volume of $10 \mu \mathrm{l}$. RT-PCR assays were performed using a CFX384 Real-Time PCR System (Bio-Rad Laboratories, Hercules, CA, USA).

\section{Western blot analysis}

The presence of YAP was determined by Western blotting. Total protein was extracted from cells using a cell lysis buffer (10× RIPA; Cell Signaling Technology, Danvers, MA, USA) containing protease inhibitors (Roche Life Science). The denatured protein was run onto $7 \%$ SDS/polyacrylamide gels, and the separated proteins were transferred to PVDF membranes (Merck Millipore) and probed with the following primary antibodies: antiYAP, anti-phosphorylated YAP (Cell Signaling Technology) diluted 1:1000, and anti- $\beta$-actin peroxidase (ACTB; Sigma-Aldrich) diluted 1:25,000. Peroxidase-conjugated, species-appropriate antibody at a 1:5000 dilution was added and then detected by autoradiography using enhanced chemoluminescence (Merck Millipore). ACTB served as the loading control.

\section{Scratch wound healing migration assay}

MSCs (passages 3-6) were seeded at a density of $1 \times 10^{4}$ cells $/ \mathrm{cm}^{2}$ in a 6-well plate and allowed to grow to confluence before being scratched with a P1000 pipette tip. Cell debris was removed by washing once with $1 \mathrm{ml}$ of culture media. New culture media supplemented with $20 \mu \mathrm{M}$ DH or $10 \mu \mathrm{M}$ LPA was then added, and cells were maintained for up to 7 days concurrently with nontreated cells. The culture medium was changed every other day. Images of the closing wound were acquired on days 3,5 , and 7 by inverted microscopy. Three independent experiments were performed.

\section{Transwell migration assay}

The MSCs were treated with $20 \mu \mathrm{M}$ DH or $10 \mu \mathrm{M}$ LPA for $24 \mathrm{~h}$ before being seeded into the insert chamber of an $8-\mu \mathrm{m}$ pore size transwell (Corning) filled with DMEM supplemented with $2 \%(\mathrm{v} / \mathrm{v}) \mathrm{FBS}, 100 \mathrm{U} / \mathrm{ml}$ penicillin, and $100 \mu \mathrm{g} / \mathrm{ml}$ streptomycin. The lower chamber contained $600 \mu$ l of completed DMEM medium (10\% FBS). The culture was then maintained at $37^{\circ} \mathrm{C}$ in a humidified atmosphere containing $5 \% \mathrm{CO}_{2}$ for $6 \mathrm{~h}$ to allow cell migration. After 6-h incubation, the numbers of cells that migrated to the other side of the transwell inserts were determined by Hoechst-33342 staining. Data are presented as the mean \pm standard error of the mean (SEM) of three independent experiments.

\section{Osteogenic differentiation}

Cells at passages 3-6 were used to study osteogenic differentiation capacity. For osteogenic differentiation, MSCs at a density of $5 \times 10^{4}$ cells were plated into a 35$\mathrm{mm}$ tissue culture dish. NH OsteoDiff ${ }^{\oplus}$ Medium (Miltenyi Biotec, Bergisch Gladbach, Germany) was then added for osteogenic differentiation induction. After 2 weeks of culture, the cells were fixed with $4 \%$ formaldehyde for $15 \mathrm{~min}$ and stained with $40 \mathrm{mM}$ Alizarin red S 
(Sigma Aldrich) for $20 \mathrm{~min}$ at room temperature (RT) to evaluate calcium deposition in the cells. Alizarin red $\mathrm{S}$ staining was quantitated by adding $10 \%$ acetic acid to the cells with subsequent incubation at RT for $30 \mathrm{~min}$ with shaking. The cells in $10 \%$ acetic acid were collected using a cell scraper, transferred into a $1.5-\mathrm{ml}$ microcentrifuge tube, and incubated at $85^{\circ} \mathrm{C}$ for $10 \mathrm{~min}$. The cell mixture was centrifuged for $15 \mathrm{~min}$ at $20,000 \mathrm{~g}$. The supernatant was collected, and the $\mathrm{pH}$ value was adjusted to 4.1-4.5 with $10 \%$ ammonium hydroxide. Sample aliquots of $50 \mu \mathrm{l} /$ well were prepared in triplicate in a 96-well plate to read the absorbance at $405 \mathrm{~nm}$ on a spectrophotometer (BioTek Instruments, Inc., Winooski, VT, USA). Alizarin red S deposited in the cells was calculated from the concentration of Alizarin red $\mathrm{S}$ relative to a standard curve.

\section{Adipogenic differentiation}

For adipogenic differentiation, MSCs at passages 3-6 were used; $5 \times 10^{4}$ cells of MSCs were cultured in $\mathrm{NH}$ AdipoDiff ${ }^{\oplus}$ Medium (Miltenyi Biotec) for 3 weeks. Cells were stained with $0.5 \%(\mathrm{w} / \mathrm{v})$ Oil Red O (Sigma Aldrich) in isopropanol for $30 \mathrm{~min}$ at RT to determine the lipid droplet in the cells. For the quantification of Oil Red O staining, the dye was eluted with $100 \%$ isopropanol by incubating cells with isopropanol for $10 \mathrm{~min}$ at RT. Solution aliquots of $200 \mu \mathrm{l} /$ well were transferred into a 96well plate to read the absorbance at $510 \mathrm{~nm}$ by spectrophotometer (BioTek). Oil Red O concentration was calculated relative to a standard curve.

\section{Sample preparation for imaging by FTIR}

Cells were trypsinized into single cells, and a drop of $4 \times 10^{5}$ cells was deposited onto IR transparent $2-\mathrm{mm}$ thick barium fluoride windows, air dried, and washed several times with distilled water to eliminate culture medium contamination before storage in a desiccator until spectra were acquired.

\section{FTIR microspectroscopy analysis}

FTIR spectra comprises three important regions, including the protein region $\left(1700-1500 \mathrm{~cm}^{-1}\right.$ : amide I and amide II protein), the lipid region $\left(3000-2800 \mathrm{~cm}^{-1}: \mathrm{CH}\right.$ stretching, $\mathrm{C}=\mathrm{O}$ ester lipid $\left(1750-1700 \mathrm{~cm}^{-1}\right.$, and the carbohydrate and nucleic acid region $\left(1300-900 \mathrm{~cm}^{-1}\right.$ : $\mathrm{P}=\mathrm{O}$ phosphates, $\mathrm{C}-\mathrm{O}, \mathrm{C}-\mathrm{C}$ glygogen, and carbohydrate $\left(1300-900 \mathrm{~cm}^{-1}\right)$. In this study, spectrum data were acquired at an infrared microspectroscopy beamline (BL4.1 IR Spectroscopy and Imaging) at the Synchrotron Light Research Institute with a Vertex 70 FTIR Spectrometer (Bruker Optics Ltd., Ettlingen, Germany) coupled with an IR microscope (Hyperion 2000; Bruker Optics Ltd.). The detector of the infrared microscope was a liquid nitrogen-cooled mercury cadmium telluride (MCT-A) detector (of $100 \mu \mathrm{m}$ in size). Measurements were performed using an aperture size of $10 \times 10 \mu \mathrm{m}$ with a spectral resolution of $6 \mathrm{~cm}^{-1}$, with 64 scans co-added over the measurement range from 4000 to $800 \mathrm{~cm}^{-1}$. Spectral acquisition and instrument control were performed using OPUS 7.5 software (Bruker Optics Ltd). Spectral changes in the functional groups were evaluated at the integral area of each peak, especially the region of amide I protein $\left(1700-1600 \mathrm{~cm}^{-1}\right)$, amide II protein (1600$\left.1500 \mathrm{~cm}^{-1}\right), \mathrm{CH}$ stretching from lipid $\left(3000-2800 \mathrm{~cm}^{-1}\right)$, $\mathrm{C}=\mathrm{O}$ ester lipid $\left(1750-1700 \mathrm{~cm}^{-1}\right)$, and $\mathrm{PO} 43-$ or nucleic acid $\left(1200-900 \mathrm{~cm}^{-1}\right)$. Spectra from each sample group were analyzed using principal component analysis (PCA). Data were preprocessed by performing a baseline correction, and they were normalized using extended multiplicative signal correction using spectral regions from 3000 to $2800 \mathrm{~cm}^{-1}$ and $1800-900 \mathrm{~cm}^{-1}$ using Unscrambler 10.1 software (CAMO Software, Oslo, Norway).

\section{Results}

Effect of pharmacological molecules on YAP

MSC samples that were characterized by immunophenotypic profiling (Additional file 1: Figure S1) were used in this study. To determine whether YAP was expressed in MSCs, we first determined the expression of YAP across cell types, including iPSCs, HEK293, and MSCs (Additional file 2: Figure S2). The results showed YAP to be highly expressed in MSCs and that it could be used as a model for studying the effect of small molecules on MSC differentiation. To confirm the efficacy of the pharmacological molecules, cells treated with $20 \mu \mathrm{M} \mathrm{DH}$ or $10 \mu \mathrm{M}$ LPA were harvested for Western blot analysis to determine YAP activity. An increase in phosphorylated YAP (p-YAP; inactive form) was observed in DHtreated cells (Fig. 1a, b), and an increase in YAP active form was found in LPA-treated cells (Fig. 1c, d). These results indicated that the pharmacological molecules were working in the expected and appropriate manner.

\section{Effect of DH and LPA on human MSC proliferation and migration}

To determine whether pharmacological molecule treatment affects hMSC proliferation and migration, in vitro wound healing assay and transwell migration assay were performed. The result of the wound healing experiment showed that the wound area completely sealed on day 7 in the LPA-treated group, while cells treated with $\mathrm{DH}$ showed delayed effect compared to control (Fig. 2a). To further confirm that DH suppresses cell migration, we performed transwell migration assay. The result showed that DH-treated cells barely migrated to the bottom side, whereas LPA was found to enhance migration (Fig. 2b). Cell count analysis confirmed that a significantly less number of cells migrated to the bottom side of the 

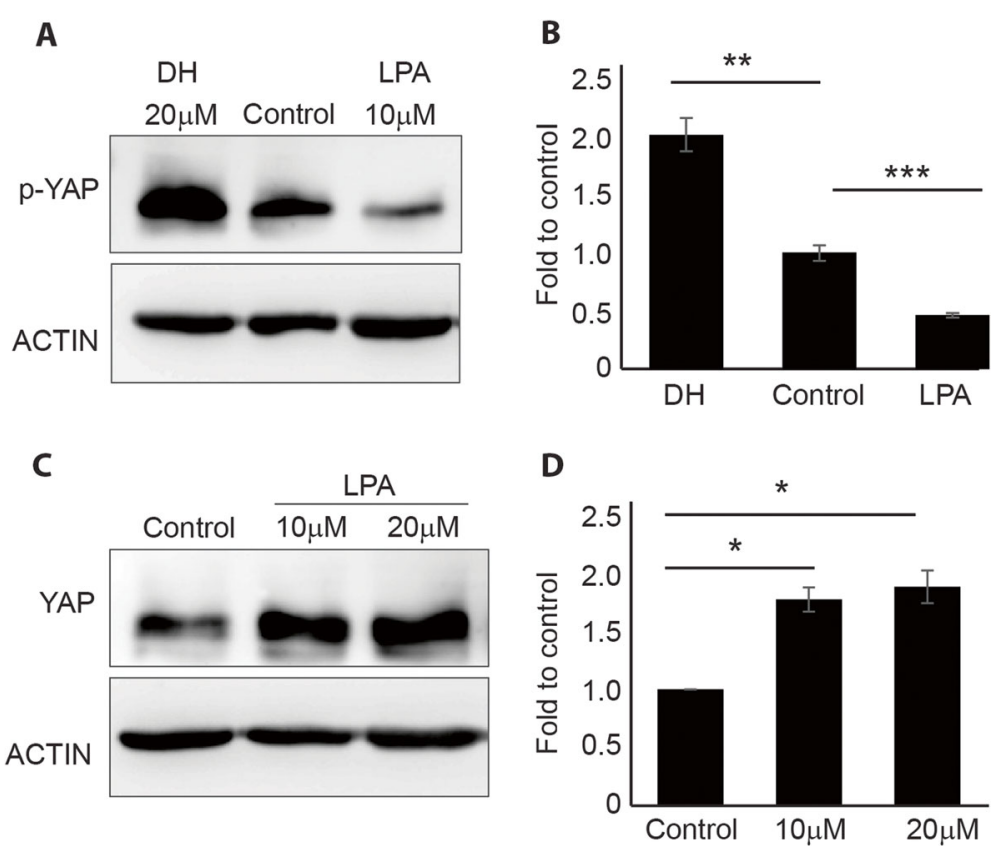

Fig. 1 Effects of DH and LPA on the expression of YAP. Western blot analysis showed the phosphorylated form of YAP in MSCs after treatment with $20 \mu M$ DH or $10 \mu \mathrm{M}$ LPA (a). Quantification of p-YAP protein levels confirmed a significant increase in p-YAP expression in DH and decreased p-YAP expression in LPA-treated cells (b). An increase in YAP upon treatment with LPA (c) and quantification of YAP protein levels confirmed a significant increase in YAP upon LPA treatment (d). Band intensity was normalized to that of actin (mean $\pm \mathrm{SEM} ; n=3,{ }^{*} P<0.05,{ }^{* *} P<0.01,{ }^{* * *} P<0.001$, Student's $t$ test)

upper chamber, as shown in Fig. 2c. Taken together, these results suggest that DH suppresses cell proliferation and migration, whereas the treatment of cells with LPA showed effects opposite to those treated by DH. To determine whether DH treatment induces cell death, cells at day 5 of treatment were collected for total cell count and to determine the number of living and dead cells using trypan blue exclusion assay. We found a significantly increased number of cells (living + dead cells) in the LPA-treated group compared to other conditions, whereas the DH-treated group had a significantly lower total number of cells. However, the number of dead cells did not differ significantly among treatments (Fig. 2d). These results indicate that DH inhibits MSC proliferation and migration, but it does not significantly induce cell death.

DH inhibits osteogenic differentiation and promotes fatforming process of human MSCs, but the inverse was observed in LPA

To determine whether the pharmacological molecules DH and LPA influence the differentiation capacity of MSCs, we performed osteoblast-like cell differentiation by culturing MSCs in cytokine-induced osteogenic differentiation medium in the presence of either LPA or DH (Fig. 3a). After 2 weeks of culture, we found that DH treatment inhibited the osteogenic differentiation of MSCs, while LPA promoted MSC differentiation to osteoblast-like cells, as demonstrated by Alizarin red staining (Fig. 3a). Quantitative measurement of calcium deposition from Alizarin red staining showed a significant decrease in calcium content in DH-treated MSCs (Fig. 3b). These results suggest that the expression level of YAP, which is a target protein of DH and LPA, could influence the differentiation capacity of MSCs.

The effects of pharmacological molecules were also investigated during the fat-forming process of MSCs. In contrast to osteogenic differentiation, DH-treated cells showed increased intracellular fat droplets compared to both control and LPA-treated cells (Fig. 3c). Quantitative measurement of fat droplets from Oil Red $\mathrm{O}$ staining showed a significant increase in fat content in DH-treated MSCs (Fig. 3d). These results suggest that the expression of YAP influences the differentiation of human MSCs.

\section{Gain- and loss-of-function experiments confirm the role of YAP in adipo-osteogenic differentiation}

To further confirm that YAP plays a critical role in osteogenic differentiation, we performed gain- and lossof-function experiments by generating YAP-knockdown (YAP-KD) and YAP-overexpressing (YAP-O/E) MSCs. The knockdown and overexpression efficiency rates were determined, as shown in Fig. 4a. Later, YAP-KD and YAP$\mathrm{O} / \mathrm{E}$ MSCs were subjected to osteogenic and adipogenic differentiation medium. Consistent with the results of small molecule treatment, YAP-KD cells exhibited less osteogenic differentiation phenotype when compared to 


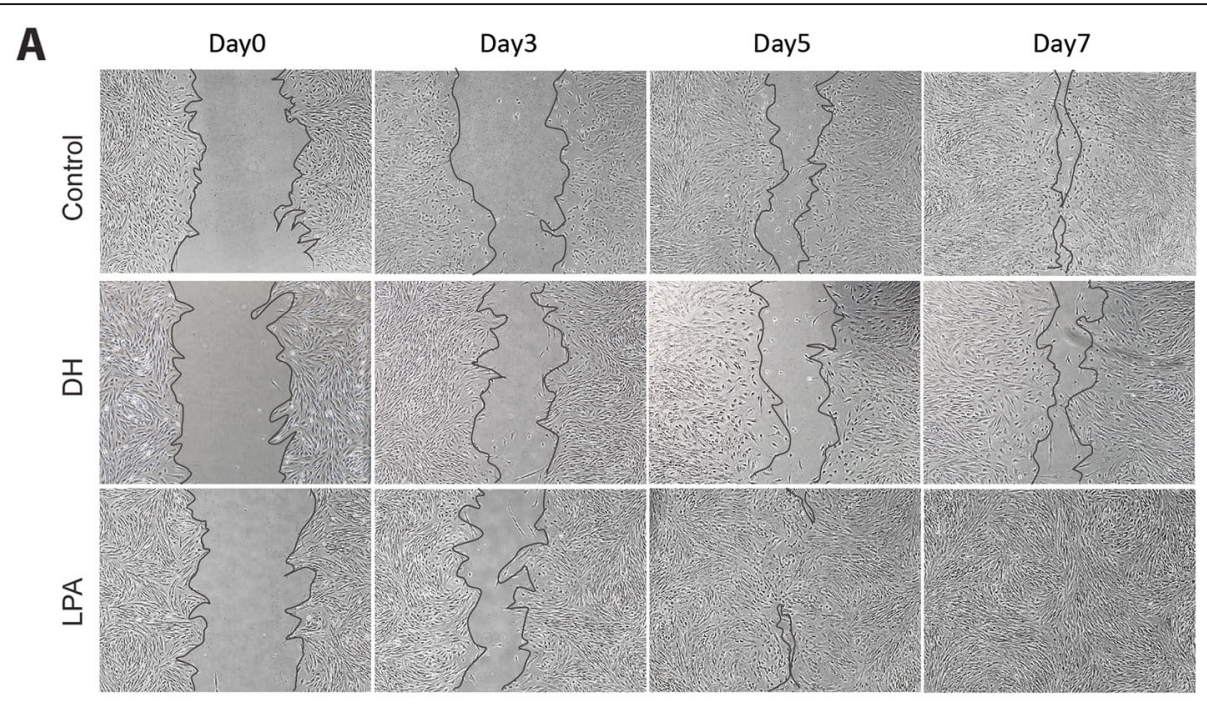

B
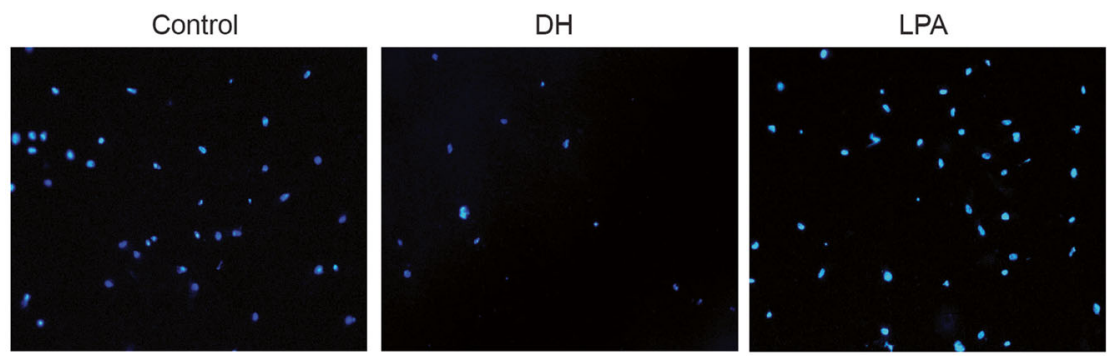

C

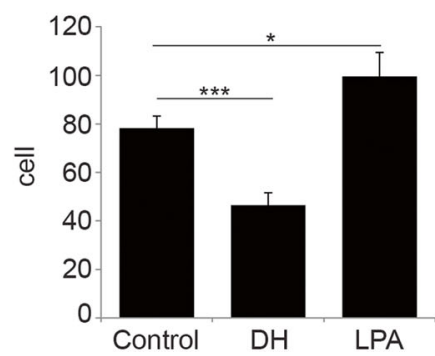

D

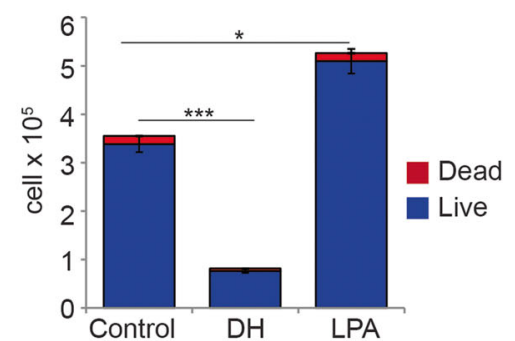

Fig. 2 Effects of DH and LPA on MSC proliferation and migration. A scratch was made using a P1000 pipette tip and cultured in the presence of DH or LPA for 7 days. The distance between edges was monitored under a microscope to determine the proliferation rate upon treatment (a). Representative pictures of cells at the bottom side of the inserted chamber after 6-h incubation stained with Hoechst-33342 (b). The number of migrated cells was counted and reported as mean \pm SEM; $n=3,{ }^{*} P<0.05,{ }^{* * *} P<0.001$, Student's $t$ test (c). MSC numbers were enumerated after treatment with DH and LPA with trypan blue exclusion (d). Statistical analysis for live cells is shown as mean $\pm S E M ; n=3,{ }^{*} P<0.05,{ }^{* * *} P<0.001$, Student's $t$ test. Numbers of dead cells were not significantly different among treatments

those of YAP-O/E and control cells (Fig. 4b, c). In contrast, YAP-depleted cells demonstrated an increased bias towards adipogenic lineage commitment than YAPoverexpressing cells, as shown in Fig. 4d, e. These results confirm that YAP plays a crucial role in both adipogenic and osteogenic differentiations.

\section{FTIR signatures of YAP-depleted MSC-derived adipocytes and osteoblasts}

To further confirm that the expression of YAP could influence the differentiation potential of hMSCs to bone and fat, differentiated cells were collected and subjected to FTIR analysis. The mean FTIR spectra, PCA analysis, and loading information of the control MSCs, YAP-O/E, and YAP-KD cells differentiated to adipocytes and osteoblasts recorded from more than 200 single cells in the mid-IR region of $4000-800 \mathrm{~cm}^{-1}$ are shown in Fig. 5 . For differentiation to osteoblasts, PCA was performed on the second derivative spectra from control MSC, YAP-O/E, and YAP-KD (Fig. 5a). Three groups of spectra were clearly separated into 2D PCA score plots: PC1 and PC2 (Fig. 5b). PC1 and PC2 explained 28\% and 20\% 


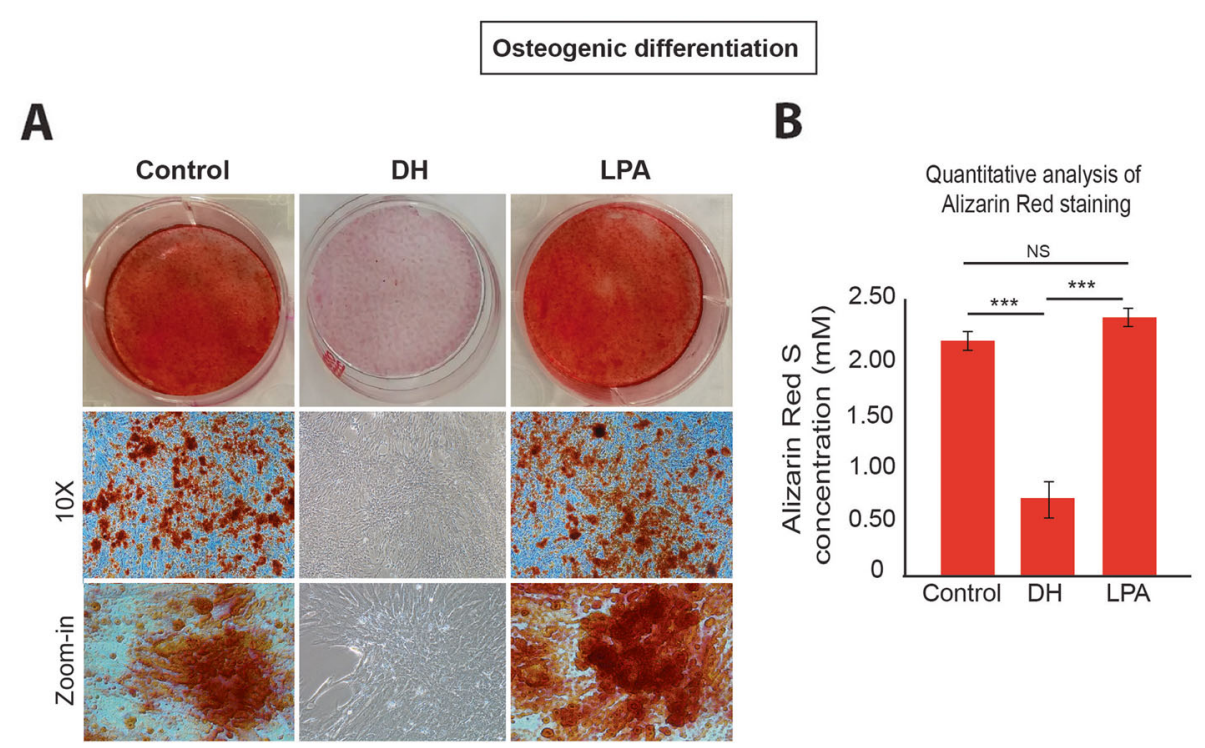

Adipogenic differentiation

C

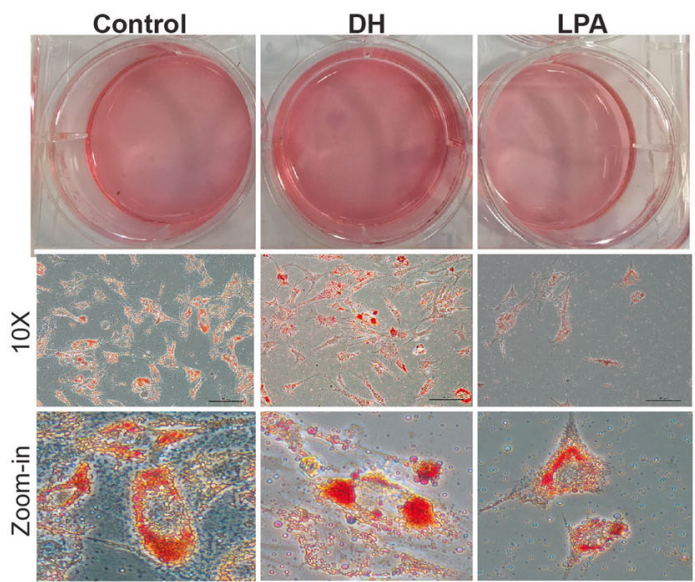

D

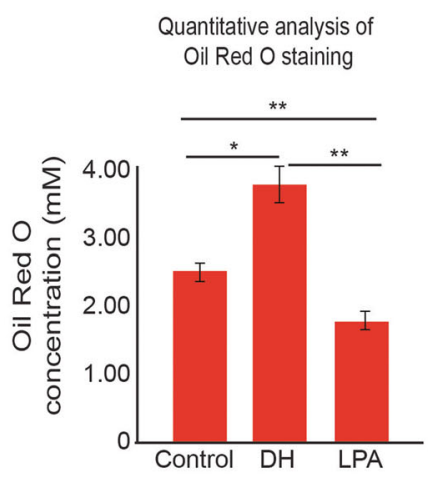

Fig. 3 Effects of DH and LPA on osteogenic and adipogenic differentiation. Alizarin red staining shows calcium content (a) and quantitative determination of Alizarin red staining (b) in MSCs after treatment with LPA or DH. Oil Red O staining shows lipid droplets in cells after treatment with LPA or DH (c) and quantitative data (d). Quantitative data are presented as mean $\pm \mathrm{SEM} ; n=3,{ }^{*} P<0.05,{ }^{* *} P<0.01$, ${ }^{* * *} P<0.001$, Student's $t$ test. NS, no significant difference

of the total variance, respectively. The spectrum groups of YAP-KD were associated with positive scores on the score plot (PC1) and presented as a negative loading of PC1. The high negative loading for PC1 loading at 1029, 1122 , and $1241 \mathrm{~cm}^{-1}$ assigned to $\mathrm{PO}_{4}{ }^{3-}$ phosphate bands, and nucleic acid from phosphodiester bonds (centered at $1241 \mathrm{~cm}^{-1}$ ) was responsible for distinguishing the YAP-KD cells from control and YAP-O/E cells (Fig. 5c). The YAP-O/E group was clearly separated along the negative score plot of $\mathrm{PC} 2$. The negative value of PC2 loading at 2931 and $2857 \mathrm{~cm}^{-1}$ corresponding to the $\mathrm{C}-\mathrm{H}$ stretching bands, $1226 \mathrm{~cm}^{-1}$ associated with phosphodiester bonds from the nucleic acid, and $\mathrm{PO}_{4}{ }^{3-}$ phosphate bands centered at $977 \mathrm{~cm}^{-1}$ and $1000 \mathrm{~cm}^{-1}$ (Fig. 5c).

Unique FTIR spectra were also found in adipogenic differentiation. The second derivative spectra from control MSC, YAP-O/E, and YAP-KD cells that differentiated to adipocytes are shown in Fig. 5d. The PCA score plot demonstrated the separation of these samples with a total variance of $33 \%$ for $\mathrm{PC} 1$ and $16 \%$ for $\mathrm{PC} 2$ (Fig. 5e). The highest positive loading plot from PC1 was 


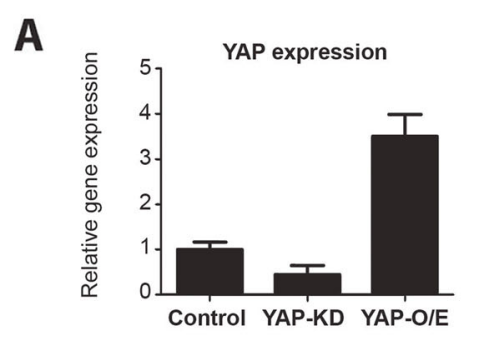

B
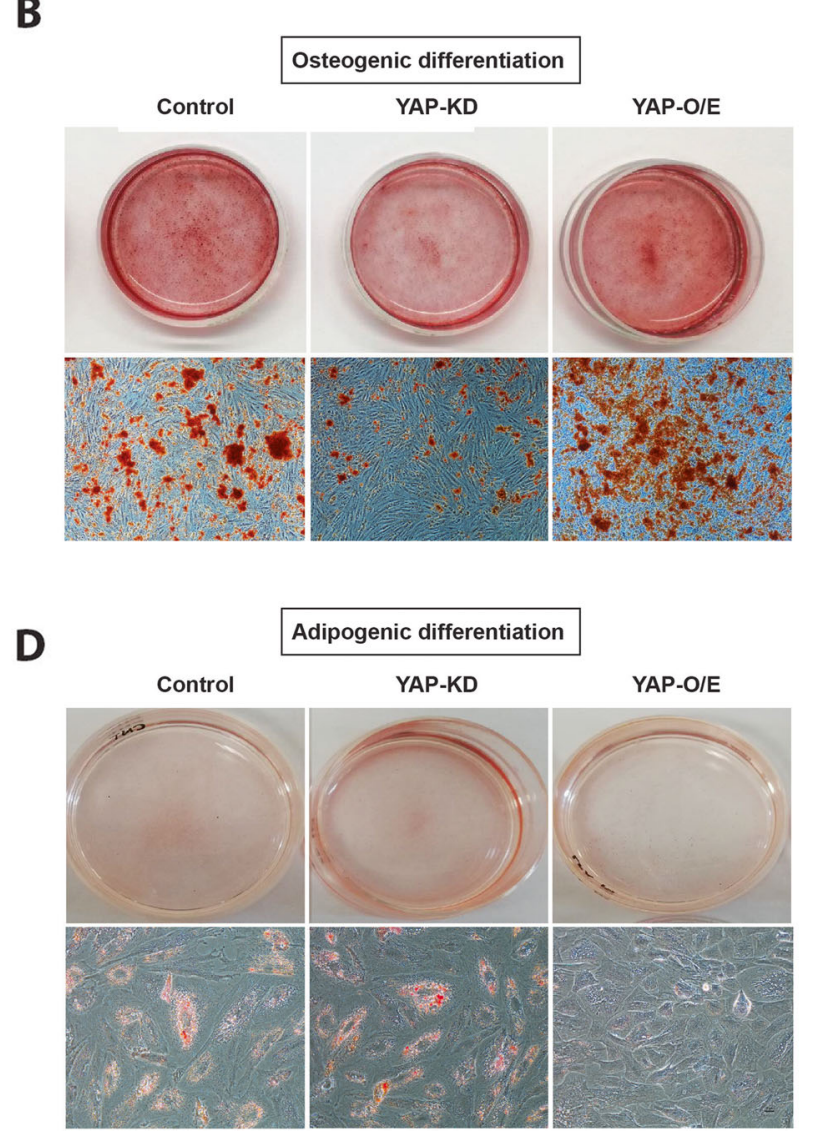

C

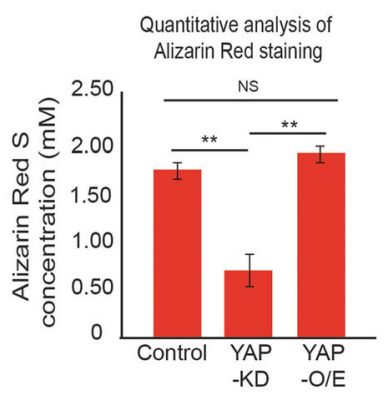

E

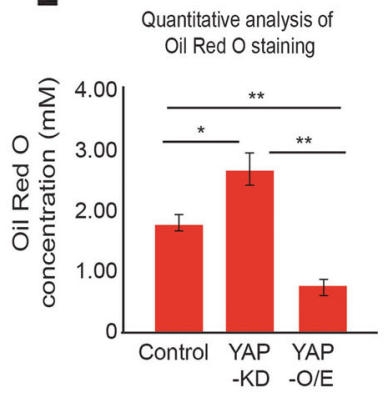

Fig. 4 Effects of gene manipulations on YAP relative to osteogenic differentiation. YAP expression level in MSCs after being forced to be downregulated and upregulated as quantitated by q-PCR (a). Alizarin red staining shows calcium content and quantitative evaluation of Alizarin red staining in control, YAP-KD, and YAP-O/E MSCS (b, c). Oil Red O staining shows lipid contents and quantification value (d, e). Quantitative data are shown as mean $\pm \mathrm{SEM} ; n=3,{ }^{*} P<0.05,{ }^{* *} P<0.01$, Student's $t$ test. NS, no significant difference

observed in the $\mathrm{CH}$ stretching $(\mathrm{CH} 2, \mathrm{CH} 3$ stretching) centered at 2921 and $2850 \mathrm{~cm}^{-1}$ from lipid, and $\mathrm{C}=\mathrm{O}$ ester lipid centered at $1741 \mathrm{~cm}^{-1}$, and oppositely correlated with negative score plot in the MSC group from the second derivative spectrum (Fig. 5f). The separation along PC2 can be explained by the positive loading for PC2 in the spectral region at 1238 and $1035 \mathrm{~cm}^{-1}$ from the nucleic acid and carbohydrate regions, respectively. The respective spectra of the YAP-O/E were associated with negative score plots in $\mathrm{PC} 2$ and presented as a positive loading of PC2 (Fig. 5f). Taken together, these results show that FTIR signatures could clearly demonstrate the consequence of YAP upon adipoosteogenic differentiation. Similarly, unique FTIR spectra were also observed in LPA- or DH-treated cells differentiated to osteoblasts and adipocytes (Additional file 3: Figure S3).

\section{Discussion}

YAP has been implicated in several types of cells for controlling cell proliferation and differentiation [29,30]. However, whether YAP plays a role in controlling adipo-osteogenic balance in humans has never been fully elucidated. In the present work, we showed that the expression level of YAP 
Osteogenic differentiation

A

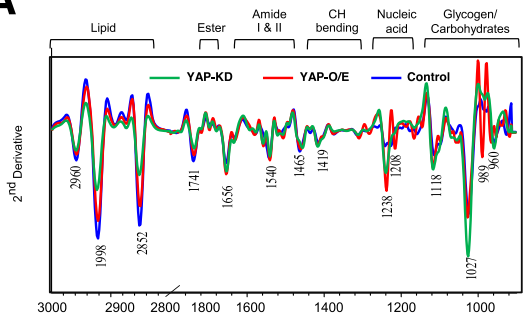

B

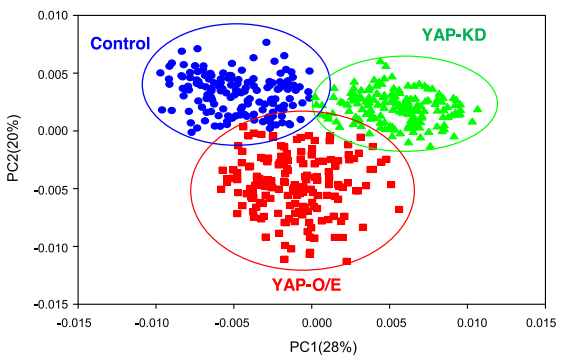

C

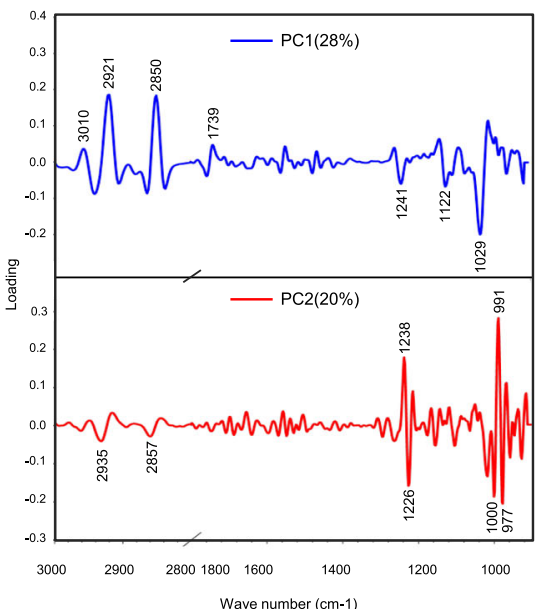

Adipogenic differentiation

D

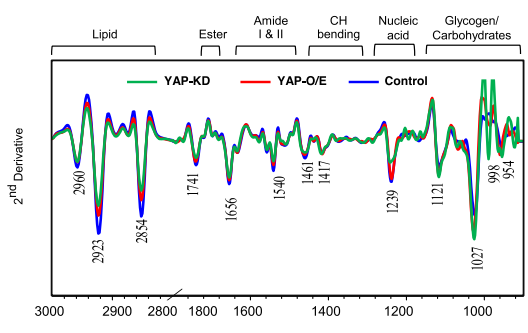

E

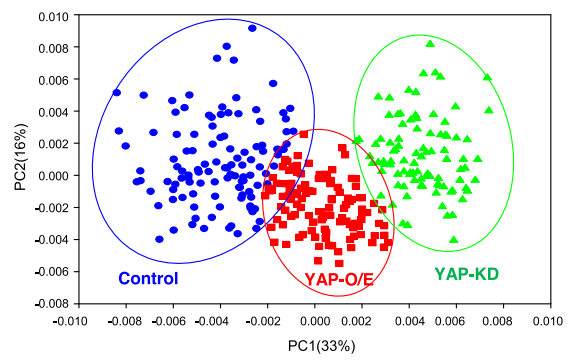

$\mathbf{F}$

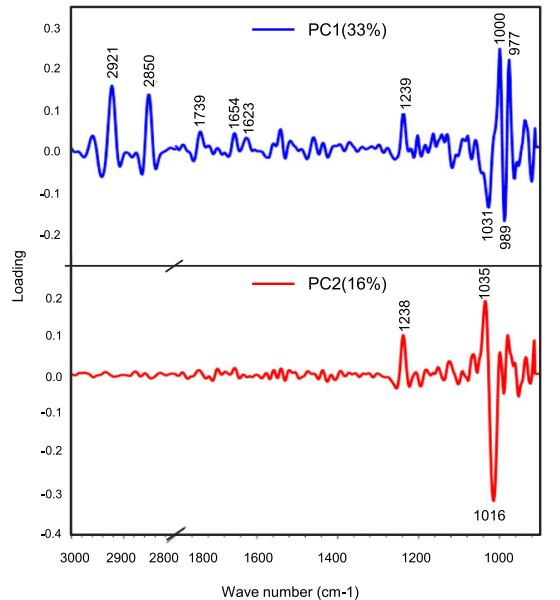

Fig. 5 FTIR signatures of YAP-depleted MSC-derived adipocytes and osteoblasts. Second derivative spectral (a, d), 2D PCA score plot of all recorded FTIR spectra of YAP-O/E, YAP-KD, and control cells (b, e). Score loading of PC1 (upper) and PC2 (lower) to identify the variable corresponding to wavelength number $(\mathbf{c}, \mathbf{f})$. Blue dots represent non-treated control, green triangles represent YAP-KD, and red squares represent YAP-O/E cells. Eclipses depicted in the plot define the confidence level with which $95 \%$ of the data are allocated

during human MSC differentiation is crucial for adipoosteogenic differentiation. Increasing YAP, either by pharmaceutical molecule or by genetic manipulation, enhances osteogenic differentiation but suppresses differentiation to adipocytes even though the cells were cultured in enriched cytokine medium that promotes and supports adipogenic differentiation. In contrast, low YAP promotes adipogenic differentiation but inhibits osteogenic differentiation. These results clearly suggest that YAP plays a crucial role in human adipo-osteogenic differentiation.

YAP is highly expressed in highly proliferative cells, such as iPSCs and cancers, since it plays a role in inducing cell proliferation and anti-apoptosis [31, 32]. Even though the Hippo-YAP/TAZ signaling pathway has been studied in pluripotent stem cells for quite some time, the role of YAP in pluripotent stem cells remains inconclusive and controversial. A study in mouse embryonic stem cells (mESCs) found that YAP is highly expressed in self-renewing mESCs but is inactivated during differentiation [33]. Overexpression of YAP inhibits mESC differentiation and maintains stem-like properties and self-renewal even under differentiation conditions. In contrast, Chung and colleagues reported that YAP is dispensable for self-renewal, but it is required for 
differentiation [29]. Interestingly, a study in human pluripotent stem cells found YAP to be irrelevant relative to maintaining pluripotency but required for selfrenewal. Overexpression of YAP could transform pluripotent stem cells into naive stem cells [30].

Aside from controlling the biological properties of pluripotent stem cells, YAP in MSCs seems to have no role in cell proliferation and anti-apoptosis processes. YAP was instead found to be crucial for the mechanosensing process by responding to the interaction of cellmatrix adhesion and by conveying the differentiation phenotype of MSCs [34-36]. In soft ECM, YAP retained in the cytoplasm undergoes a degradation process. This phenomenon enhances the likelihood of differentiation towards adipogenesis. Conversely, stiff ECM induces YAP to translocate into the nucleus to induce osteogenesis. These results clearly suggest that genes that are involved in adipo-osteogenesis are downstream targets of YAP. In this experiment, the expression of YAP was manipulated by pharmaceutical molecules and genetic approaches to determine the differentiation capacity of MSCs. Our results confirm that YAP is an essential molecule for adipo-osteogenic lineage decision of MSCs.

The mechanism by which YAP regulates adipoosteogenesis was demonstrated by Pan and colleagues [17]. They found that YAP interacts with beta-catenin to promote osteogenic differentiation and maintain bone homeostasis in a mouse model [17]. Recently, transcriptional coactivator with PDZ motif (TAZ), a YAP homo$\log$ protein, was identified in mouse MSCs (C3H10T1/2 cell line) and in human adipose tissue-derived stem cells as an important effector protein that binds to Smad4 to regulate the balance of lineage commitment in osteogenic and adipogenic differentiation. However, they found that YAP did not interact with Smad4 in either osteogenic or adipogenic differentiation of MSCs [19]. Thus, multiple mechanisms may account for YAP regulation of adipo-osteogenesis of human MSCs, which requires further investigation.

FTIR microspectroscopy has been applied in the biomedical field to study global structural and compositional changes in the nucleic acids, proteins, and lipids of many biological samples, including stem cells and differentiated cells [24, 37-42]. SR FTIR was shown to provide information that can be used as a chemical fingerprint of biological cells. It is very useful for detecting physiological changes in cells that can be related to their physiological properties. Previous studies in stem cells have shown that the structures of nucleic acid, protein, and lipid have changed along the differentiation time course $[24,38,41,42]$ and that these changes result in a shift in the corresponding peaks in the FTIR spectra. Hence, the dynamics of the peaks during differentiation may have the potential for use as biomarkers to identify the stage of the cell. Moreover, FTIR microspectroscopy has a high signal-to-noise ratio of less than $10 \mu \mathrm{m}$ per spot on the sample and enhanced diffraction-limited lateral spatial resolution. These advantages suggest the possibility that FTIR microspectroscopy can be used to identify even a small difference in a cell at the single-cell level. So far, this technique can be applied to study stem cell differentiation. The spectral signature of cells can be applied to create a spectral database of stem cell differentiation that will allow us to identify correlations between differentiation potential and spectral signature.

\section{Conclusions}

In this study, we demonstrated that the expression level of YAP is essential for adipo-osteogenic differentiation of human MSCs. Increasing YAP activity, either by pharmaceutical molecule or by genetic manipulation, enhances osteogenic differentiation but suppresses differentiation to adipocytes. In contrast, low YAP activity promotes adipogenic differentiation but inhibits osteogenic differentiation. These results clearly suggest that YAP plays a crucial role in human adipo-osteogenic differentiation. We also showed that FTIR can be used as an effective method for studying cell characteristics of MSC-derived osteoblasts and adipocytes.

\section{Supplementary information}

Supplementary information accompanies this paper at https://doi.org/10. 1186/s13287-019-1494-4.

\begin{abstract}
Additional file 1: Figure S1. UC-hMSCs were subjected to immunophenotypic profiling using flow-cytometry. Cells from all three cell lines (A-C) did not express CD34 or CD45, but they were positive for CD73, CD90, and CD105 expression. (PPTX $102 \mathrm{~kb}$ )

Additional file 2: Figure S2. Western blot analysis of total YAP and actin in induced pluripotent stem cells (iPSCs), HEK293, and MSCs.

Additional file 3: Figure S3. FTIR spectral signatures of MSCs treated with LPA or DH during differentiation towards osteoblasts or adipocytes. Second derivative spectral $(A, E)$, two-dimensional PCA score plot of all recorded FTIR spectra of DH, LPA, and control cells (B, F). Score loading of $P C 1(C, G)$ and $P C 2(D, H)$ to identify the variable corresponding to wavelength number. Blue dots represent non-treated control, green triangles represent $\mathrm{DH}$, and red squares represent LPA treated cells. Eclipses depicted in the plot define the confidence level with which $95 \%$ of the data are allocated. (PPTX $450 \mathrm{~kb}$ )
\end{abstract}

\section{Abbreviations}

MSCs: Mesenchymal stem cells; YAP: Yes-associated protein; UC: Umbilical cord blood; FTIR: Fourier transform infrared microspectroscopy; Runx: RUNX family transcription factor 2; PPAR: Peroxisome proliferator-activated receptor Y; PEPCK: Phosphoenolpyruvate carboxykinase; ALBP: Adipocyte lipid-binding protein; DH: Dobutamine hydrochloride; LPA: Lysophosphatidic acid; KD: Knockdown; O/E: Overexpression

\section{Acknowledgements}

The authors gratefully acknowledge the volunteers who donated tissues for this study, the staff of the maternity wards at Siriraj Hospital that facilitated the specimen collection, and Prof. Davor Solter and Prof. Barbara Knowles for their valuable suggestions. 


\section{Authors' contributions}

$\mathrm{CL}$ conceived and designed the experiments, analyzed the data, and wrote the paper. KTh designed and analyzed the synchrotron data. KTa, NJ, and ND performed the experiments. $C L$ and $Y U$ analyzed the data. SI supervised the project and edited the paper. All authors read and approved the final version of the manuscript.

\section{Funding}

This study was supported by a grant from the Faculty of Medicine Siriraj Hospital, Mahidol University; a grant from the Thailand Research Fund to $\mathrm{CL}$ (grant no. RSA-6080089) and to SI (grant no. RTA 488-0007); and a Commission on Higher Education Grant to SI (grant no. CHE-RES-RG-49). ND and SI were supported by the grant from the Royal Golden Jubilee (RGJ) Scholarship (PHD/0102/2559), Thailand Research Fund.

\section{Availability of data and materials}

All datasets in this article are included within the article and additional files.

\section{Ethics approval and consent to participate}

This study was approved by the ethics committee of the Siriraj Institutional Review Board (COA no. Si101/2015) of the Faculty of Medicine Siriraj Hospital, Mahidol University, Bangkok, Thailand. This study complied with the principles set forth in the Declaration of Helsinki, the Belmont Report, the CIOMS Guidelines, and the ICH-GCP. Umbilical cord blood (UC) was obtained from healthy newborns after written informed consent to do so was obtained from their mothers.

\section{Consent for publication}

Not applicable

\section{Competing interests}

The authors declare that they have no competing interests.

\section{Author details}

${ }^{1}$ Siriraj Center of Excellence for Stem Cell Research, Department of Medicine, Faculty of Medicine Siriraj Hospital, Mahidol University, 2 Wanglang Road, Bangkoknoi, Bangkok 10700, Thailand. 'Synchrotron Light Research Institute (Public Organization), Nakhon Ratchasima, Thailand. ${ }^{3}$ Department of Immunology, Faculty of Medicine Siriraj Hospital, Mahidol University, Bangkok, Thailand. ${ }^{4}$ Division of Hematology, Department of Medicine, Faculty of Medicine Siriraj Hospital, Mahidol University, Bangkok, Thailand. ${ }^{5}$ Bangkok Hematology Center, Wattanosoth Hospital, BDMS Center of Excellence for Cancer, Bangkok, Thailand.

Received: 1 August 2019 Revised: 28 October 2019 Accepted: 13 November 2019 Published online: 18 December 2019

\section{References}

1. Kern S, Eichler H, Stoeve J, Kluter H, Bieback K. Comparative analysis of mesenchymal stem cells from bone marrow, umbilical cord blood, or adipose tissue. Stem Cells. 2006;24:1294-301.

2. Ankrum JA, Ong JF, Karp JM. Mesenchymal stem cells: immune evasive, not immune privileged. Nat Biotechnol. 2014;32:252-60.

3. Li XY, Bai JP, Ji XF, Li RG, Xuan YL, Wang YM. Comprehensive characterization of four different populations of human mesenchymal stem cells as regards their immune properties, proliferation and differentiation. Int J Mol Med. 2014;34:695-704.

4. Chen Q, Shou P, Zheng C, Jiang M, Cao G, Yang Q, Cao J, Xie N, Velletri T, Zhang $X, X u C$, Zhang $L$, et al. Fate decision of mesenchymal stem cells: adipocytes or osteoblasts? Cell Death Differ. 2016;23:1128-39.

5. Ge CX, Cawthorn WP, Li Y, Zhao GS, Macdougald OA, Franceschi RT. Reciprocal control of osteogenic and adipogenic differentiation by ERK/MAP kinase phosphorylation of Runx2 and PPAR transcription factors. J Cell Physiol. 2016;231:587-96.

6. Picke AK, Campbell GM, Schmidt FN, Busse B, Rauner M, Simon JC, Anderegg U, Hofbauer LC, Saalbach A. Thy-1 deficiency augments bone loss in obesity by affecting bone formation and resorption. Front Cell Dev Biol. 2018;6127. https://doi.org/10.3389/fcell.2018.00127.

7. Li W, Xu P, Wang CZ, Ha XD, Gu YJ, Wang Y, Zhang J, Xie JX. The effects of fat-induced obesity on bone metabolism in rats. Obes Res Clin Pract. 2017: 11:454-63.
8. Cao JJ. Effects of obesity on bone metabolism. J Orthop Surg Res. 2011;6:30. https://doi.org/10.1186/1749-799X-6-30.

9. Coelho MJ, Cabral AT, Fernandes MH. Human bone cell cultures in biocompatibility testing. Part I: osteoblastic differentiation of serially passaged human bone marrow cells cultured in alpha-MEM and in DMEM. Biomaterials. 2000;21:1087-94.

10. Coelho MJ, Fernandes MH. Human bone cell cultures in biocompatibility testing. Part II: effect of ascorbic acid, betaglycerophosphate and dexamethasone on osteoblastic differentiation. Biomaterials. 2000;21:1095-102.

11. Halder G, Dupont S, Piccolo S. Transduction of mechanical and cytoskeletal cues by YAP and TAZ. Nat Rev Mol Cell Biol. 2012;13:591-600.

12. Chen $Q$, Shou PS, Zhang LY, Xu CL, Zheng CX, Han YY, Li WZ, Huang Y, Zhang XR, Shao CS, Roberts Al, Rabson AB, et al. An osteopontin-integrin interaction plays a critical role in directing adipogenesis and osteogenesis by mesenchymal stem cells. Stem Cells. 2014;32:327-37.

13. Tormos KV, Anso E, Hamanaka RB, Eisenhart J, Joseph J, Kalyanaraman B, Chandel NS. Mitochondrial complex III ROS regulate adipocyte differentiation. Cell Metab. 2011;14(4):537-44. https://doi.org/10.1016/..cmet.2011.08.007.

14. Zur Nieden NI, Kempka G, Rancourt DE, Ahr HJ. Induction of chondro-, osteo- and adipogenesis in embryonic stem cells by bone morphogenetic protein-2: effect of cofactors on differentiating lineages. BMC Dev Biol. 2005;5:1. https://doi.org/10.1186/1471-213X-5-1.

15. Khosla S, Drake MT, Volkman TL, Thicke BS, Achenbach SJ, Atkinson EJ, Joyner MJ, Rosen CJ, Monroe DG, Farr JN. Sympathetic beta(1)-adrenergic signaling contributes to regulation of human bone metabolism. J Clin Investig. 2018;128:4832-42.

16. Bao Y, Nakagawa K, Yang Z, Ikeda M, Withanage K, Ishigami-Yuasa M, Okuno $Y$, Hata S, Nishina $H$, Hata Y. A cell-based assay to screen stimulators of the Hippo pathway reveals the inhibitory effect of dobutamine on the YAPdependent gene transcription. J Biochem. 2011;150:199-208.

17. Pan JX, Xiong L, Zhao K, Zeng P, Wang B, Tang FL, Sun D, Guo HH, Yang X, Cui $S$, Xia WF, Mei $L$, et al. YAP promotes osteogenesis and suppresses adipogenic differentiation by regulating beta-catenin signaling. Bone Res. 2018;6:18. https://doi.org/10.1038/s41413-018-0018-7.

18. Pan JX, Xiong L, Zhao K, Zeng P, Wang B, Yang X, Xiong WC. YAP promotes osteogenesis and suppresses adipogenic differentiation. J Bone Miner Res. 2017;32:S229.

19. Park JS, Kim M, Song NJ, Kim JH, Seo D, Lee JH, Jung SM, Lee JY, Lee J, Lee YS, Park KW, Park SH. A reciprocal role of the Smad4-Taz axis in osteogenesis and adipogenesis of mesenchymal stem cells. Stem Cells. 2019;37:368-81.

20. Simon MF, Daviaud D, Pradere JP, Gres S, Guigne C, Wabitsch M, Chun J, Valet P, Saulnier-Blache JS. Lysophosphatidic acid inhibits adipocyte differentiation via lysophosphatidic acid 1 receptor-dependent downregulation of peroxisome proliferator-activated receptor gamma 2. J Biol Chem. 2005:280:14656-62.

21. Ami D, Natalello A, Mereghetti P, Neri T, Zanoni M, Monti M, Doglia SM, Redi CA. FT-IR spectroscopy supported by PCA-LDA analysis for the study of embryonic stem cell differentiation. Spectrosc Int J. 2010;24:89-97.

22. Ami D, Neri T, Natalello A, Mereghetti P, Doglia SM, Zanoni M, Zuccotti M, Garagna S, Redi CA. Embryonic stem cell differentiation studied by FT-IR spectroscopy. Biochim Biophys Acta Mol Cell Res. 2008;1783:98-106.

23. Heraud $\mathrm{P}$, Tobin MJ. The emergence of biospectroscopy in stem cell research. Stem Cell Res. 2009;3:12-4.

24. Krafft C, Salzer R, Seitz S, Ern C, Schieker M. Differentiation of individual human mesenchymal stem cells probed by FTIR microscopic imaging. Analyst. 2007;132:647-53.

25. Luanpitpong S, Janan M, Thumanu K, Poohadsuan J, Rodboon N, Klaihmon $P$, Issaragrisil S. Deciphering the elevated lipid via CD36 in mantle cell lymphoma with bortezomib resistance using synchrotron-based Fourier transform infrared spectroscopy of single cells. Cancers (Basel). 2019;11(4). https://doi.org/10.3390/cancers 11040576.

26. Baker MJ, Trevisan J, Bassan P, Bhargava R, Butler HJ, Dorling KM, Fielden PR, Fogarty SW, Fullwood NJ, Heys KA, Hughes C, Lasch P, et al. Using Fourier transform IR spectroscopy to analyze biological materials. Nat Protoc. 2014; 9:1771-91.

27. Dumas $P$, Miller $L$. The use of synchrotron infrared microspectroscopy in biological and biomedical investigations. Vib Spectrosc. 2003;32:3-21.

28. Heraud P, Ng ES, Caine S, Yu QC, Hirst C, Mayberry R, Bruce A, Wood BR, McNaughton D, Stanley EG, Elefanty AG. Fourier transform infrared 
microspectroscopy identifies early lineage commitment in differentiating human embryonic stem cells. Stem Cell Res. 2010;4:140-7.

29. Chung H, Lee BK, Uprety N, Shen W, Lee J, Kim J. Yap1 is dispensable for self-renewal but required for proper differentiation of mouse embryonic stem (ES) cells. EMBO Rep. 2016;17:519-29.

30. Qin H, Hejna M, Liu Y, Percharde M, Wossidlo M, Blouin L, DurruthyDurruthy J, Wong P, Qi Z, Yu J, Qi LS, Sebastiano V, et al. YAP induces human naive pluripotency. Cell Rep. 2016;14:2301-12.

31. Johnson R, Halder G. The two faces of Hippo: targeting the Hippo pathway for regenerative medicine and cancer treatment. Nat Rev Drug Discov. 2014; 13:63-79.

32. Chan SW, Lim CJ, Chen L, Chong YF, Huang C, Song H, Hong W. The Hippo pathway in biological control and cancer development. J Cell Physiol. 2011; 226:928-39.

33. Lian I, Kim J, Okazawa H, Zhao J, Zhao B, Yu J, Chinnaiyan A, Israel MA, Goldstein LS, Abujarour R, Ding S, Guan KL. The role of YAP transcription coactivator in regulating stem cell self-renewal and differentiation. Genes Dev. 2010;24:1106-18.

34. Mohri Z, Del Rio HA, Krams R. The emerging role of YAP/TAZ in mechanotransduction. J Thorac Dis. 2017;9:E507-9.

35. Dupont $\mathrm{S}$. Role of YAP/TAZ in cell-matrix adhesion-mediated signalling and mechanotransduction. Exp Cell Res. 2016;343:42-53.

36. Dupont S, Morsut L, Aragona M, Enzo E, Giulitti S, Cordenonsi M, Zanconato F, Le Digabel J, Forcato M, Bicciato S, Elvassore N, Piccolo S. Role of YAP/ TAZ in mechanotransduction. Nature. 2011;474:179-83.

37. Thumanu K, Tanthanuch W, Ye DN, Sangmalee A, Lorthongpanich C Parnpai R, Heraud P. Spectroscopic signature of mouse embryonic stem cell-derived hepatocytes using synchrotron Fourier transform infrared microspectroscopy. J Biomed Opt. 2011;16(5):057005. https://doi.org/10. $1117 / 1.3580253$

38. Tanthanucha W, Thumanu K, Lorthongpanich C, Parnpai R, Heraud P. Neura differentiation of mouse embryonic stem cells studied by FTIR spectroscopy. J Mol Struct. 2010;967:189-95.

39. Bentley AJ, Nakamura T, Hammiche A, Pollock HM, Martin FL, Kinoshita S, Fullwood NJ. Characterization of human corneal stem cells by synchrotron infrared micro-spectroscopy. Mol Vis. 2007;13:237-42.

40. Liu ZX, Tang YZ, Chen F, Liu X, Liu ZJ, Zhong JJ, Hu J, Lu JH. Synchrotron FTIR microspectroscopy reveals early adipogenic differentiation of human mesenchymal stem cells at single-cell level. Biochem Biophys Res Commun. 2016;478:1286-91.

41. Chonanant C, Jearanaikoon N, Leelayuwat C, Limpaiboon T, Tobin MJ, Jearanaikoon P, Heraud P. Characterisation of chondrogenic differentiation of human mesenchymal stem cells using synchrotron FTIR microspectroscopy. Analyst. 2011;136:2542-51.

42. Ward DF, Salasznyk RM, Klees RF, Backiel J, Agius P, Bennett K, Boskey A, Plopper GE. Mechanical strain enhances extracellular matrix-induced gene focusing and promotes osteogenic differentiation of human mesenchymal stem cells through an extracellular-related kinase-dependent pathway. Stem Cells Dev. 2007;16:467-79.

\section{Publisher's Note}

Springer Nature remains neutral with regard to jurisdictional claims in published maps and institutional affiliations.

Ready to submit your research? Choose BMC and benefit from:

- fast, convenient online submission

- thorough peer review by experienced researchers in your field

- rapid publication on acceptance

- support for research data, including large and complex data types

- gold Open Access which fosters wider collaboration and increased citations

- maximum visibility for your research: over $100 \mathrm{M}$ website views per year

At BMC, research is always in progress.

Learn more biomedcentral.com/submissions 